MS03-P03

\section{Long-wavelength phasing at the P13 EMBL Macromolecular Crystallography beamline at Petra III}

Guillaume Pompidor ${ }^{1}$, Gleb Bourenkov ${ }^{1}$, Isabel Bento ${ }^{1}$, Johanna

Hakanpää ${ }^{1}$, Ivars Karpics ${ }^{1}$, Thomas Schneider ${ }^{1}$

1. European Molecular Biology Laboratory, Hamburg, Germany

email: pompidor@embl-hamburg.de

The P13 Macromolecular Crystallography beamline, operated by the EMBL at PETRA III, enables diffraction data collection over a wide energy range, from $4 \mathrm{keV}$ to 17.5 $\mathrm{keV}(0.7-3.1 \AA)[1]$. In order to fully exploit the beamline capability at low energy $\left(10^{12} \mathrm{ph} \cdot \mathrm{s}^{-1}\right.$ at $\left.5 \mathrm{keV}\right)$, the PILATUS $6 \mathrm{M}-\mathrm{F}$ detector, with a $450 \mathrm{~mm}$ Si sensor thickness and with a custom calibration at low energy, is mounted on a 2 q-angle stage to increase the maximum resolution achievable $(2.4 \AA$ at $4 \mathrm{keV})$.

We will present and describe the typical experimental setup for long wavelength data collection. For low-energy experiments, typically below $5.5 \mathrm{keV}$, the air absorption can be drastically reduced by the use of a helium path. The gas is circulating inside the detector and in a homemade cone, which can be fixed on the detector upon user request. The beamline can be setup for low-energy data collection in about 10 minutes and remains fully compatible with the use of the sample changer, for high-throughput screening, and the mini-k of the MD2 diffractometer allowing the reorientation of the crystal for optimized anomalous data collection.

Several examples of structure determination using intrinsic anomalous scatterers, such as sulfur (SAD) or calcium (MAD at the $\mathrm{Ca} K$-edge at $4.05 \mathrm{keV}$ ) will be presented. We will especially demonstrate the efficiency of the He-path in the improvement of diffraction data quality at low energy.

To extend the experimental opportunities, a small laboratory dedicated to the preparation of derivatives is available to users. With more than 150 compounds available, the heavy atom library contains many elements $(\mathrm{Cd}, \mathrm{Pd}, \mathrm{U} \ldots)$ exhibiting strong anomalous signal at low energy, which are excellent candidates for SAD and MAD experiment at long wavelength.

References:

[1] Cianci, M. et al., (2017). Journal of synchrotron radiation 24, 323-332.

Keywords: Long-wavelength phasing
MS03-P04

\section{Simultaneous XRD and XES at XFELs, elucidating electronic and geometric structures of metalloproteins}

Roberto Alonso Mori ${ }^{1}$

1. SLAC National Accelerator Laboratory, Menlo Park, United States of America

email: robertoa@slac.stanford.edu

One of the fields where the advantages of X-ray free electron lasers (XFELs) have proven to be very fruitful is in the study of metalloproteins(1). These proteins, where metal centers efficiently catalyze chemical processes under ambient conditions, are ubiquitous in nature. X-Ray diffraction (XRD) has become a standard technique in synchrotron radiation (SR) sources worldwide to elucidate the geometric structure of crystallized proteins, and X-ray spectroscopic techniques to provide information about the electronic structure of the active site. Despite the enormous progress of the field achieved from SR-based measurements, these studies suffer from some experimental constraints, the main being the radiation-induced damage of the sample by the $\mathrm{X}$-rays, which makes it difficult to collect the signal from the intact sample for radiation-sensitive systems.

At XFELs, each fs X-ray pulse is short enough to probe the sample and yield a detectable signal outrunning the SR-type radiation damage (in the ps) scale. The consequence is the possibility to measure sensitive systems under ambient, and therefore, functional conditions. The short X-ray pulses also allow the study of these processes in real time by following the catalytic reactions and the associated electronic and geometric changes step by step with fs time resolution. In this presentation I will describe the methods developed in the past few years at XFELs to study metalloproteins and discuss some examples and applications. In particular I will emphasize how X-ray emission spectroscopy can be used as an in situ method for monitoring the integrity of metal catalytic centers during simultaneous XRD data collection(2).

References:

1-R. Alonso-Mori and J. Kern. (2017) "X-ray Free Electron Lasers". Chapter VII "Damage-Free Electronic and Geometric Structure Determination of Metalloproteins". Royal Society of Chemistry 2-R. Alonso-Mori, J. Kern, R.J. Gildea et al. (2012) "EnergyDispersive X-ray Emission Spectroscopy Using an X-ray Free Electron Laser In a Shot-by-Shot mode". Proc. Natl. Acad. Sci. U.S.A. 109, 19103-19107

Keywords: XES, Spectroscopy, XFEL 